\title{
The multiple contributions of thyroid hormone to heat production
}

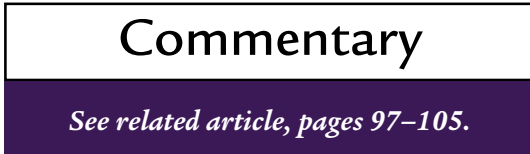

\author{
J. Enrique Silva \\ Department of Medicine, Division of Endocrinology, Jewish General Hospital, McGill University, \\ Montreal, Quebec H3T 1E2, Canada. Phone: (514) 340-7550; Fax: (514) 340-7529; E-mail: enrique.silva@staff.mcgill.ca. \\ J. Clin. Invest. 108:35-37 (2001). DOI:10.1172/JCI200113397.
}

The advent of homeothermy during evolution brought with it the need of mechanisms to produce heat to maintain body temperature in environments usually colder than the body. Thermogenic mechanisms are customarily classified as either obligatory or facultative. Obligatory thermogenesis (OT) represents the energy dissipated as heat in the many energetic transformations inherent to life. As discussed below, OT may be higher in warmblooded species, possibly reflecting a lower thermodynamic efficiency of homeothermic machine compared with the poikilothermic machine. Facultative, or adaptive, thermogenesis (FT) is the additional heat production that the body activates in response to cold or overfeeding. The magnitude of OT determines the thermoneutrality temperature - the environmental temperature at which FT is activated, at which point neither heat-producing nor heat-dissipating mechanisms are activated, and body temperature is maintained solely on the basis of OT.

The thyroid gland is present in all vertebrate species, and the hormones produced by its follicular epithelium (collectively, thyroid hormone, $\mathrm{TH}$ ) play important roles in development, the most dramatic and well-known example being amphibian metamorphosis. Warm-blooded species are unique, however, in that TH increases oxygen consumption and stimulates enzymes, such as the mitochondrial 3-phosphate glycerol dehydrogenase and lipogenic enzymes (1), that may be accessory to their thermogenic effect. That TH increases the rate of aerobic metabolism and heat production has been known for over a century (2), but we still do not have a clear understanding of this physiological role of the thyroidal secretion.

\section{$\mathrm{TH}, \mathrm{FT}$, and brown adipose tissue}

For a long time it was assumed that TH stimulated only OT. The recogni- tion that it also plays an essential role in FT is recent and has gradually evolved, starting with the realization that brown adipose tissue (BAT) and its unique mitochondrial uncoupling protein (UCP) are central to FT in mammals. BAT of hypothyroid rats fails to respond to cold with an increase in guanosine di phosphate (GDP) binding to its mitochondria (a measure of UCP concentration and activation); but, while this failure and the associated hypothermia can be readily corrected by administering physiological doses of $\mathrm{T}_{4}$, higher doses of this hormone are, surprisingly, associated with reduced, rather than further increased, GDP binding (3). These observations led to the idea that $\mathrm{TH}$ played only a permissive role in thermogenesis by BAT. This permissive role, it later emerged, was more apparent than real. First, sympathetic stimulation of BAT bears an inverse relationship to the thyroid status (ref. 4 and references therein). Secondly, the BAT content of the most active $\mathrm{TH}, \mathrm{T}_{3}$, is strongly influenced by the type II iodothyronine $5^{\prime}$ deiodinase (D2), which is activated by the sympathetic nervous system and inhibited powerfully by its substrate, $T_{4}$ (5). In hypothyroidism, BAT will be highly stimulated but will be less responsive because of the lack of TH. In hyperthyroidism, conversely, BAT will be understimulated, an effect that is compounded by the reduction in D2 activity resulting from both the lower sympathetic activity and the excess of $\mathrm{T}_{4}$.

Based on this understanding of the physiological regulation of $\mathrm{D} 2$, it became apparent that UCP levels vary dramatically with $T_{3}$ concentration in BAT and that D2 effectively provides the $T_{3}$ necessary for a maximal thermogenic response to adrenergic stimulation $(6,7)$. This led ultimately to the finding that $T_{3}$ directly and independently stimulates UCP gene tran- scription in the rat, as does cAMP, and that these two stimuli synergistically enhance the activity of the UCP promoter (reviewed in ref. 8). The findings by Ribeiro et al. (9), published in this issue of the JCI, bring additional strong support to the concept the TH plays an essential role in FT and demonstrate such a role in another species, the mouse, which had been questioned (10).

\section{Distinct roles for the $\alpha$ and $\beta$ TH receptor isoforms}

From a wider perspective, Ribeiro et al. (9) provide further support to the concept that multiple mechanisms have been selected through evolution for $\mathrm{TH}$ to stimulate heat production. They confirm that $\mathrm{TH}$ contributes to BAT thermogenesis in two ways: by stimulating the expression of critical elements of the norepinephrine (NE) signaling pathway and by inducing the UCP directly. They further demonstrate that $\mathrm{TH}$ action on a limiting element of the NE signaling pathway is mediated by the $\alpha_{1}$ TH receptor (TR) isoform, while the $\beta T R$ is sufficient to stimulate UCP. However, in view of the absolute requirement for UCP in the thermogenic response (11) and the absence of a thermogenic defect in $\beta T R$-deficient mice (12), it appears that, whereas the $\alpha_{1}$ TR isoform is indispensable for the NE signaling, the $\beta T R$ isoform is not strictly required to induce UCP stimulation. It should be noted that most of the work defining the TH response elements in the UCP gene employed cells transfected with $\beta_{1} \mathrm{TR}$ (13), the most abundant isoform of $\beta$ TR. Therefore, it would now be of interest to repeat those studies in cells expressing only the $\alpha_{1}$ TR.

The selective or preferential use of a $\mathrm{TR}$ isoform by TH is not unprecedented. The $\beta$ TR seems to play a an essential role in TSH regulation and cochlear development (14), while $\alpha \mathrm{TR}$ 
seems to be more important in mediating the chronotropic and inotropic effects of TH on the heart $(15,16)$. The lower body temperature of $\alpha_{1}$ TRknockout $\left(\alpha_{1}\right.$ TR-KO), but not $\beta$ TR$\mathrm{KO}$, mice $(15,16)$ appears to implicate the $\alpha_{1}$ TR in the thermogenic effect of TH. Following up on this observation, Ribeiro et al. (9) now show that $\alpha \mathrm{TR}$ is essential for $\mathrm{TH}$ to restore the levels of a factor in the NE signaling pathway, downstream of the adrenergic receptors, that is limiting in hypothyroidism. They observe that when hypothyroid rats are treated with the $\beta$ TR-selective analogue GC1 (9), not only does cAMP generation in BAT remain impaired, but the animals' heart rate fails to increase, in contrast to animals treated with $\mathrm{T}_{3}$. Thus, the $\alpha_{1}$ TR isoform appears to be important for NE signaling in other tissues as well. In turn, this finding suggests that the impaired NE signaling is at the core of the thermoregulatory deficit in the $\alpha_{1}$ TR-deficient mice, a conclusion that is further supported by the fact that FT remains impaired in hypothyroid mice treated with GC1, even though the analogue restores normal levels of UCP. One might argue that the lower body temperature of the $\alpha_{1}$ TR-KO mice at room temperature is also due to the sympathetic deficiency. Since $22-23^{\circ} \mathrm{C}$ ("room temperature") is below thermoneutrality for small rodents $\left(28-30^{\circ} \mathrm{C}\right)$, mice maintained at $22^{\circ} \mathrm{C}$ will need to activate FT to maintain their core temperatures. This reasoning, however, does not exclude the participation of $\alpha_{1}$ TR in the maintenance of OT, as discussed below.

\section{The importance of thermodynamic inefficiency in thermogenesis}

Paradoxically, in spite of the comparatively recent awareness of the role of $\mathrm{TH}$ in FT, we know more of the mechanisms involved in the activation of this form of thermogenesis than in that of OT. BAT thermogenesis shows us that reducing the thermodynamic efficiency of biological processes may subserve the purpose of generating heat to maintain body temperature. Is it possible that such a "strategy" had been selected as well to enhance OT? The answer is probably yes, and $\mathrm{TH}$ is likely to play a central role. Consider a poikilothermic animal (for example, a lizard), placed at the thermoneutrality temperature of a mammal of the same size (e.g., a mouse; $30^{\circ} \mathrm{C}$ ). While the lizard will rapidly equilibrate its body temperature with the ambient, the mouse will remain homeothermic and without activating FT, since by definition is at thermoneutrality temperature. We can infer from this simple observation that OT is higher in the mouse than in the lizard, and indeed the mammal has a faster metabolic rate, both globally and on an organ-byorgan basis (17). Moreover, other findings suggest that the increased heat production is due, at least in part, to a lower thermodynamic efficiency of the mammal (18) and not merely to a larger number of energy transactions in the mammalian machine. Interestingly enough, comparison of hypothyroid and euthyroid rat muscle shows that, for any given amount of mechanical work, the euthyroid muscle generates more heat (19). Similarly, euthyroid hepatocytes spend more ATP to generate the same amount of glycogen from lactate than hypothyroid hepatocytes (20). Therefore, TH seems to reduce the thermodynamic efficiency of the warm-blooded machine for the sake of producing heat. An analysis of possible mechanisms suggested that the stimulation of ATP turnover, reflecting more "metabolic business," can only explain about $50 \%$ of the thermogenic effect of TH (21). Harper and Brand arrived at the same conclusion studying hepatocytes from hypo-, eu-, and hyperthyroid rats (22) and proposed that the difference was accounted for by an uncoupling of ATP synthesis via a regulated proton leak, much in the same way UCP uncouples phosphorylation in BAT. The cloning of UCP2 and UCP3 genes brought the hope that their products might explain the thermogenic effect through a $\mathrm{TH}$-induced reduction in the thermodynamic efficiency of ATP generation. However, the targeted disruption of the corresponding genes does not seem to support such expectation $(23,24)$.

While some of the molecular mechanisms whereby $\mathrm{TH}$ stimulates OT remain unaccounted for , the article by Ribeiro et al. (9) invites speculation on the extent to which the $\alpha \mathrm{TR}$ could mediate metabolic effects of TH. As mentioned above, one can argue that the half-a-degree-lower core temperature of the $\alpha 1$ TR-deficient mouse (16) is due to a reduced FT caused by a limiting $\alpha 1$ TR-dependent factor in the
NE signaling pathway. However, the extent to which FT participates in maintaining body temperature at room temperature (about $22^{\circ} \mathrm{C}$ ) has not been defined. UCP-null mice apparently do not show hypothermia at room temperature, and their sensitivity to cold was revealed by the challenge of exposing them at $5^{\circ} \mathrm{C}(11)$. It is therefore conceivable that FT does not play a major role in maintaining temperature in mildly cold environments; hence, the mild hypothermia of the $\alpha_{1}$ TR-null mice may be caused to a significant extent by reduced OT. It would be interesting to examine these mice at thermoneutrality as well under blockade of the sympathetic nervous system to define the role of a putative sympathetic impairment in their thermoregulatory insufficiency. Furthermore, it would be of interest to examine other responses to adrenergic stimulation, such as lipolysis and gluconeogenesis, to determine to what extent the $\alpha 1 \mathrm{TR}$ isoform is involved in lipolysis or other metabolic effects of $\mathrm{TH}$ that could be necessary for the thermogenic response.

\section{Potential clinical implications}

There are multiple potential clinical implications of the findings by Ribeiro et al. (9) and the emerging concept of $\mathrm{TR}$ isoform-selective responses to $\mathrm{TH}$. Further analysis is needed of the TR isoform selectivity of the multiple effects of TH. The availability of an $\alpha_{1}$ TR-selective agonist may allow a direct test to determine which of TH's effects are specific to this receptor isoform and to investigate the sources of variability of the responses to $\mathrm{TH}$ in thyroid dysfunction. The development of additional isoform-specific agonists and antagonists would clearly be useful in this regard. One might even be able to induce the effects of TH selectively, activating thermogenesis, for example, without concomitantly stimulating appetite and lipogenesis, which counteract the energy dissipation caused by TH. We have recently shown that an individual's resting energy expenditure is very sensitive to minor variations in the availability of $\mathrm{TH}$ (25), yet we know that thyroid cancer patients do not show weight loss in response to the mild hyperthyroidism we purposely induce with $\mathrm{L}^{-\mathrm{T}_{4}}$ to maintain plasma thyroid stimulatin factor (TSH) reduced. This indicates 
that the increased appetite and lipogenesis compensate for the increase in energy expenditure. It makes sense that the thermogenic effect of TH has been coupled to stimulation of appetite and lipogenesis during evolution; otherwise, the advent of the thermogenic effect of $\mathrm{TH}$ would have increased energy demands without supplying metabolic energy to meet them. It might now be helpful for the treatment of obesity if we could dissociate these effects.

1. Weirich, R.T., Schwartz, H.L., and Oppenheimer, J.H. 1987. An analysis of the interrelationship of nuclear and plasma triiodothyronine in the sea lamprey, lake trout, and rat: evolutionary considerations. Endocrinology. 120:664-677.

2. Magnus-Levy, A. 1895. Ueber den respiratorischen Gaswechsel unter Einfluss de Thyroidea sowie unter verschiedenen pathologische Zustand. Berlin Klin. Wochschr. 32:650-652.

3. Triandafillou, J., Gwilliam, C., and HimmsHagen, J. 1982. Role of thyroid hormone in coldinduced changes in rat brown adipose tissue mitochondria. Can. J. Biochem. 60:530-537.

4. Silva, J.E. 2000. Catecholamines and the sympathoadrenal system in thyrotoxicosis. In Werner and Ingbar's The Thyroid: a fundamental and clinical text. L.E. Braverman and R.D. Utiger, editors. Lippincott Williams \& Wilkins. Baltimore, Maryland, USA. 642-651.

5. Silva, J.E., and Larsen, P.R. 1986. Interrelationships among thyroxine, growth hormone, and the sympathetic nervous system in the regulation of 5 -iodothyronine deiodinase in rat brown adipose tissue. J. Clin. Invest. 77:1214-1223.
6. Bianco, A.C., and Silva, J.E. 1987. Intracellular conversion of thyroxine to triiodothyronine is required for the optimal thermogenic function of brown adipose tissue. J. Clin. Invest. 79:295-300.

7. Bianco, A.C., and Silva, J.E. 1987. Optimal response of key enzymes and uncoupling protein to cold in brown adipose tissue depends on local $\mathrm{T}_{3}$ generation. Am. J. Physiol. 253:E255-E263.

8. Silva, J.E., and Rabelo, R. 1997. Regulation of the uncoupling protein gene expression. Eur. J. Endocrinol. 136:251-264.

9. Ribeiro, M.O., et al. 2001. Thyroid hormone-sympathetic interaction and adaptive thermogenesis are thyroid hormone receptor isoform-specific. $J$. Clin. Invest. 108:97-105.

10. Rehnmark, S., et al. 1989. Brown adipocytes differentiated in vitro can express the gene for the uncoupling protein thermogenin: effects of hypothyroidism and norepinephrine. Exp. Cell Res. 182:75-83.

11. Enerbäck, S., et al. 1997. Mice lacking mitochondrial uncoupling protein are cold-sensitive but not obese. Nature. 387:90-94.

12. Weiss, R.E., et al. 1998. Thyroid hormone action on liver, heart, and energy expenditure in thyroid hormone receptor beta-deficient mice. Endocrinology. 139:4945-4952.

13. Rabelo, R., Schifman, A., Rubio, A., Sheng, X., and Silva, J.E. 1995. Delineation of thyroid hormone responsive sequences within a critical enhancer in the rat uncoupling protein gene. Endocrinology. 136:1003-1013.

14. Forrest, D., Erway, L.C., Ng, L., Altschuler, R., and Curran, T. 1996. Thyroid hormone receptor beta is essential for development of auditory function. Nat. Genet. 13:354-357.

15. Johansson, C., Gothe, S., Forrest, D., Vennstrom, B., and Thoren, P. 1999. Cardiovascular phenotype and temperature control in mice lacking thyroid hormone receptor- $\beta$ or both $\alpha 1$ and $\beta$. Am J. Physiol. 276:H2006-H2012.

16. Wikstrom, L., et al. 1998. Abnormal heart rate and body temperature in mice lacking thyroid hormone receptor alpha 1. EMBO J. 17:455-461.

17. Else, P.L., and Hulbert, A.J. 1981. Comparison of the "mammal machine" and the "reptile machine": energy production. Am. J. Physiol. 240:R3-R9.

18. Woledge, R.C. 1989. Energy transformations in living muscle. In Energy transformations in cells and organisms. W. Wieser and E. Gnaiger, editors. Georg Thieme Verlag, Stuttgart. New York, New York, USA. 36-45.

19. Leijendekker, W.J., van Hardeveld, C., and Elzinga, G. 1987. Heat production during contraction in skeletal muscle of hypothyroid mice. Am. J. Physiol. 253:E214-E220.

20. Berry, M.N., et al. 1989. The thermodynamic regulation of cellular metabolism and heat production. In Energy transformations in cells and organisms. W. Wieser and E. Gnaiger, editors. Georg Thieme Verlag, Stuttgart. New York, New York, USA. 18-27.

21. Silva, J.E. 1995. Thyroid hormone control of thermogenesis and energy balance. Thyroid. 5:481-492.

22. Harper, M.E., and Brand, M.D. 1993. The quantitative contributions of mitochondrial proton leak and ATP turnover reactions to the changed respiration rates of hepatocytes from rats of different thyroid status. J. Biol. Chem. 268: $14850-14860$.

23. Gong, D.W., et al. 2000. Lack of obesity and normal response to fasting and thyroid hormone in mice lacking uncoupling protein-3. J. Biol. Chem. 275:16251-16257.

24. Arsenijevic, D., et al. 2000. Disruption of the uncoupling protein-2 gene in mice reveals a role in immunity and reactive oxygen species production. Nat. Genet. 26:435-439.

25. Al-Adsani, H., Hoffer, L.J., and Silva, J.E. 1997. Resting energy expenditure is sensitive to small dose changes in patients on chronic thyroid hormone replacement. J. Clin. Endocrinol. Metab. 82:1118-1125. 